\title{
Privacy and anonymity in public sharing of high-dimensional datasets: legal and ethical restrictions
}

Jacob Jolij $(1,2 *)$, Els van Maeckelberghe $(3,4)$, Rosalie Koolhoven (5), Monicque M. Lorist $(2,4,6)$

1. Department of Research Technology and Development, Faculty of Behavioral and Social Sciences, University of Groningen

2. Department of Experimental Psychology, Faculty of Behavioral and Social Sciences, University of Groningen, The Netherlands

3. Institute for Medical Education, University Medical Center Groningen, The Netherlands

4. Sprint@Work, Groningen, The Netherlands

5. Department of Law and Information Technology, Faculty of Law, University of Groningen, The Netherlands

6. Neuroimaging Center (NIC), University of Groningen, The Netherlands

*) Corresponding author:

Dr. J. Jolij

Department of Research Technology and Development

Faculty of Behavioral and Social Sciences

University of Groningen

Grote Kruisstraat 2/1

9712 TS Groningen, The Netherlands

+31503636348

j.jolii@rug.nl 


\begin{abstract}
Over the past years, psychology has seen a remarkable move to increasing research transparency, following several high-profile cases of scientific misconduct and the realization that reproducibility of psychological research findings may be disappointingly low. Taking from exact science disciplines such as experimental physics, there is increasing support for public sharing of empirical data, with some researchers even arguing that public data sharing should be a requirement for publication. Indeed, the principle of data transparency is a core value in science. However, in psychology and cognitive neuroscience, researchers work with human subject data. This makes that data sharing is subject to legal and ethical restrictions. Here we discuss the legal and ethical implications of public data sharing, as advocated by several authors. We conclude that large scale public sharing leads to inevitable legal and ethical problems with participant privacy, especially in the light of continuous technological developments. What might be 'anonymity' today could be become personal data tomorrow.
\end{abstract}




\section{Introduction}

Over the past years, it has become increasingly clear that there are severe problems with the accessibility, replicability, and reproducibility of results in psychology and cognitive neuroscience. Psychological science has seen high profile controversies surrounding the publication of highly improbable results ${ }^{1}$ and even outright fraud. The case of Diederik Stapel may easily be the most notorious cases of fraud in academia of the past decades ${ }^{2}$. Stapel admitted to have fabricated data underlying no less than 54 of his academic publications, including papers in high profile journals such as Science. Strong claims have been made that a large portion of published research may, in fact, be false ${ }^{3}$. Indeed, the results of the Psychology Reproducibility Project, a large scale replication attempt of a number of key studies in psychology, suggest that the reproducibility rate of psychological studies may be as low as $34 \%{ }^{4}$. Although a large scale replication study for cognitive neuroscience has not been conducted yet, there is reason to assume that the replication rate for the more "brain-oriented" areas of psychology is only marginally, if any, better than for psychology ${ }^{5}$.

The causes of these substantial problems with reproducibility have been attributed to problems with statistical inference, (over)interpretation of results, publication bias, publication pressure, and lack of accountability of researchers. Reforms, at the level of governments, granting agencies, research institutions, and scientific journals are slowly introduced in order to turn the tide. One important aspect of these reforms is ensuring the availability of data underlying published empirical papers. Obviously, sharing data is a fundamental aspect of any empirical science. However, several authors have signaled problems with data sharing in the field of psychology ${ }^{6} ;{ }^{78}$. The availability of data for validation, reanalysis, or secondary analyses appears to be limited.

To remedy this situation, sharing of research data is increasingly encouraged, for example by granting agencies, journals, and individual researchers. Presently, there is considerable discussion amongst scientists, amongst others via social media, about best practices in data sharing, with a small but vocal minority strongly advocating public data sharing, and pushing Ethics Committees to allow or even enforce public data sharing (see the discussion listed at https://www.facebook.com/groups/psychmap/search/?query=informed\%20consent for several examples). Public sharing of scientific data is not new, and is in fact commonplace in many of the exact sciences. Data from CERN experiments, for example, is live-streamed to a publicly accessible archive where anyone can analyze the data, and NASA regularly releases data such as photos of interplanetary 
missions into the public domain. Psychologists and cognitive neuroscientists, however, work with human subject data. Therefore, they are necessarily bound by legal and ethical restrictions with regard to sharing and publishing data. There is a general tendency to be reserved with respect to sharing data involving human subjects, and many Ethics Committees do only allow data sharing with qualified individuals (i.e., other researchers), or do not set any guidelines at all, and leave data sharing fully at the responsibility of researchers.

Although there is still quite some resilience against public data sharing within psychology, the practice is on the rise nonetheless ${ }^{9}$. Public data sharing is stimulated, for example, by providing 'open data' badges for papers of which the data is publicly available ${ }^{10}$, or even demanding data to at least be available for peer review, and ideally publicly shared ${ }^{11}$. These measures have resulted in a considerable increase in data sharing: since the flagship psychology journal Psychological Science started providing badges, the proportion of papers with openly available data increased from $3 \%$ to $39 \%{ }^{12}$.

However, it is far from clear what the exact legal and ethical implications of large scale public sharing of human subject data are, in particular for datasets containing many variables (so-called high dimensional data) such as neuroimaging data ${ }^{13}$, and genomic data. Although several authors have proposed concrete steps to move toward public sharing, or already put such methods in practice $\left({ }^{14}\right.$, there is also agreement that many of the legal and ethical aspects of publicly sharing data are yet unclear. For example, what exactly constitutes sensitive data? Or, who gets to decide what kind of data can or cannot be publicly shared? However, one of the most pressing matters regards participant confidentiality. How is participant anonymity, one of the most fundamental rights of research participants, properly safeguarded if data is shared publicly ${ }^{15}$ ?

In this paper, we will discuss several of the legal and ethical implications of publicly sharing human subject data, in particular that of high dimensional datasets, such as neuroimaging data, in particular with respect to privacy and anonymity issues. Given that most authors thus far have focused primarily on the benefits of public data sharing for science (see for example ${ }^{16} ;{ }^{11}{ }^{13}$, here we will deliberately take a position that some researchers might find extremely stringent with regard to interpretation of legal and ethical codes. However, by doing so, we hope to spark a debate on the legal and ethical implications of public data sharing. In the end, we strive to arrive at a position on how to responsibly share for reproducible science, without creating legal and ethical issues, and safeguarding the rights and 
autonomy of participants, and researchers.

\section{What constitutes sharing and 'open data'?}

Before we can properly discuss the consequences of sharing data, we first need to properly define the meaning of 'data sharing' and 'open data'. In the legal literature, a distinction is made between information and data. Any observation falls within the scope of the notion of 'data'. Information, on the other hand, is 'interpreted data' - data that was given a certain meaning as the result of interpretation

${ }^{17}$. In legal terms, one easily refers to any observation as 'data', even if the observation has no meaning to others or is not yet given a meaning by interpretation.

'Data sharing' refers to the act of making research data available to other parties, typically qualified researchers. According to some definitions, any data that is shared or available for sharing is 'open data'. However, others only apply the term 'open data' to publicly shared data, that is, data that is accessible without any constraint to any interested party ${ }^{11} ;{ }^{15}$. In this paper, we will use term 'publicly shared data' whenever discussing this latter form of data sharing in order to avoid confusion.

\section{Ethical and legal requirements regarding privacy and anonymity}

From an ethics perspective, anonymity and confidentiality are key rights of research participants which are grounded in Article 24 of the Declaration of Helsinki ${ }^{18}$ : "Every precaution must be taken to protect the privacy of research subjects and the confidentiality of their personal information". Most Institutional Review Boards, Ethics Committees, and professional organizations, have based their ethics guidelines on the Declaration of Helsinki. As a result, anonymity and confidentiality are typically a requirement strictly upheld by Institutional Review Boards and Ethics Committees. Whether such ethics guidelines are also legally binding depends on the local legal context - under Dutch law, for example, any ruling by a recognized Medical Ethics Committee is legally binding, and not meeting the conditions set by such a committee is a criminal offence ${ }^{19}$. However, most psychological research does not require medical ethics permission, and is submitted to local review boards. In such cases, institutional bylaws govern the enforcement of ethics guidelines.

Besides these guidelines, there is 'hard law'. The European legislator has recently taken up a modernization of the data protection rules with a view to the development of technology and increasing importance of data protection in society, amongst which scientific research data. 
In 2016 the European legislator adopted the Regulation (EU) 2016/679 of the European Parliament and of the Council of 27 April 2016 on the protection of natural persons with regard to the processing of personal data and on the free movement of such data, and repealing Directive 95/46/EC ${ }^{20}$. In short this set of rules is referred to as the 'General Data Protection Regulation' or GDPR (2016/679). This Regulation should be implemented in the Member States laws by May 25, 2018. The main objective of the GDPR (2016/679) is to empower the European citizen to become aware of the technology, the risks, the value of their data and to act upon that themselves. The ratio behind this approach is that data collection, analyses and use are increasingly invisible as technology develops.

Because the implementation of the GDPR 2016 differs in each European Member State and is at this very moment 'work in progress', we primarily address its ratio and goals in this paper. Readers are advised to consult own legal departments to discuss the legislative situation in and transposition of European law into their jurisdiction (if applicable).

Under European data protection law personal data are defined in Article 4 (1) as any information relating to an identified or identifiable natural person, a 'data subject'. According to Article 5 (1) GDPR personal data shall be (a) processed lawfully, fairly and in a transparent manner in relation to the data subject and (b) collected for specified, explicit and legitimate purposes and not further processed in a manner that is incompatible with those purposes. As for scientific research purposes 'further processing' in accordance with Article 89(1), shall not be considered to be incompatible with the initial purposes ('purpose limitation'). Furthermore they should be (f) processed in a manner that ensures appropriate security of the personal data, including protection against unauthorised or unlawful processing and against accidental loss, destruction or damage, using appropriate technical or organisational measures. Processing is lawful - even of very sensitive data (mentioned in Article 9 GDPR) such as sexual orientation - in the sense of Article 5 (1) (a) jo. Article 6 (1) (a) when the data subject has given consent to the processing of his or her personal data for one or more specific purposes or when ( $f$ ) processing is necessary for the purposes of the legitimate interests pursued by the controller or by a third party, except where such interests are overridden by the interests or fundamental rights and freedoms of the data subject which require protection of personal data. 
How does this relate to public data sharing? From both the ethics and legal guidelines it is obvious that researchers need to guarantee participant anonymity in order to even be allowed to share data publicly. The Declaration of Helsinki dictates that researchers should take all possible measures to protect participant confidentiality and anonymity; moreover, if data is not properly anonymized, and can be traced to individuals, even if they are not explicitly named in the dataset, the GDPR applies. Although the GDPR does provide exemptions for scientific research in article 21, paragraph 6, these exemptions do not apply for publicly shared data, since researchers cannot guarantee that such data is exclusively used for scientific research. Even if data is publicly shared under license (e.g., anyone downloading the dataset must declare they will only use the data for scientific purposes), it is still the researcher's responsibility to enforce this license, and inform data subjects of any other use of the data. This leads to an important question: when can we consider a dataset to be properly anonymized?

\section{What is anonymity?}

When research participants are promised anonymity in an informed consent, it is usually not specified what 'anonymity' entails in this context. We define anonymity in the strictest sense: when promising participants anonymity, this promise entails that published data cannot be traced back to an individual participant, no matter by whom. It is often argued that re-identification of publicly shared data is a negligible phenomenon: the number of individuals who seek to re-identify data records (or 'adversaries') is likely to be very low or unknowable ${ }^{21}$.

However, the number of potential adversaries is irrelevant with respect to anonymity, both from a legal and ethical perspective. In a ruling of 2012, the Dutch Council of State has ruled that under Dutch privacy law, personal data is defined as data relating to an identified or identifiable natural person (emphasis added) - even if only one adversary may be able to re-identify a dataset, technically this means the Privacy Protection Act applies to this dataset, as the dataset concerns personal data ${ }^{22}$.

Moreover, with regard to privacy protection, the core issue is not whether an adversary wants to reidentify a dataset, but rather an adversary is reasonably able to re-identify a dataset. Researchers have no control over the intentions and motives of potential adversaries, and should therefore assume that for any dataset, there will be someone out there willing to de-anonymize it. However, researchers do have control over the potential for re-identification of dataset. In order to ensure privacy and anonymity, it is this latter aspect that should be the focus of attention. 
Therefore, in assessing whether a dataset has been sufficiently anonymized, what one needs to consider is if it is in principle, without unreasonable effort, possible to de-anonymize a dataset, even if deanonymization is unlikely, or only possible when having auxiliary information.

\section{How anonymous are high-dimensional datasets?}

High-dimensional datasets are datasets in which a large number of variables per participant are recorded. Neuroimaging datasets are typical examples: the number of variables in raw neuroimaging data is very high (for example, all the voxels in an fMRI image).

Anonymization of any dataset is typically achieved by masking records in a database that can be used for identification. Name is an obvious example, but depending on the demographics of the sample, other variables may be used for identification as well. For example, if data is recorded about gender, age, and religion, it may be that in a sample there is only one Muslim male between 40 and 50 years old. Having this information, this person can be identified easily. In such a case, a researcher needs to mask the records that can lead to identification, in this case age and religion, and possibly even gender. This is referred to as $k$-anonymity: no database should contain records that can be identified by a unique set of recorded identifiers ${ }^{23}$. If this is the case, records need to be masked (that is, not reported in the database, such as name or address), or recoded (e.g., reporting an age range, such as 30-40, instead of reporting actual age).

However, it is immediately apparent that this can result in scenarios in which the anonymized data is effectively useless for further research, because vital records have been masked. Moreover, kanonymity may not be a good de-identification strategy for datasets containing a large number of variables, such as high-dimensional datasets. In general, the more variables are recorded, the higher the risk of re-identification. Any set of variables may be regarded as a pattern, and when sufficient variables are measured, such patterns are typically specific for individuals. Let us consider a practical example: movie preferences. In 2008, Netflix released its Netflix Prize Dataset to the public domain. This dataset contains little more than movie preferences of individual customers. This information is already sufficient to pinpoint individuals, when cross-referencing this with other publicly available information, in this case, IMDb reviewer profiles containing names ${ }^{24}$. 
To demonstrate that these problems also apply to a real case with psychological data, we show how the records of the lead author of this paper can be identified from the dataset on Big Five personality factors in a sample of 8,954 first year psychology students of the University of Amsterdam ${ }^{2526}$. This data is available from the Data Archiving and Networking Services of the Dutch Royal Academy of Arts and Sciences at https://easy.dans.knaw.nl/ui/datasets/id/easy-dataset:51655, for registered users only (in other words, technically this is not a publicly shared dataset, and the exemptions for scientific research in the GDPR apply).

The lead author participated in the test session in his freshman year, 1997; he was 18 years old at that time. This data can be (amongst other places) directly or indirectly be inferred from online published information, such as social media, or his CV (e.g., as published here: http://www.jolij.com/?page id=30). The dataset records the year in which the test was administered by specifying a 'test week' number, consecutively counting up starting at 15 (referring to 1982) to 40 (2007), the age of the participant at testing, the gender of the participant, the unique identifier of the participant, which is used to identify this participant within her/his cohort in all experiments using first year participants, and the scores on the Big Five scales, with scores ranging from 0 to 70 . Finding the records of the lead author would give us access to his responses on the individual items, but also to his unique identifier, potentially allowing us to find all his data in datasets in which he participated as first year student, and this identifier was recorded.

Auxiliary data we have readily available are the year of testing (1997), age at testing (18), and gender (male). From the 8,954 records in the database, using only this data, already 8,944 records can be eliminated - only 10 records fit this profile. However, in order to pinpoint the exact record we will need additional data. The lead author took a short, 15 minute online personality quiz via the website Truity (https://www.truity.com/test/big-five-personality-test) to obtain a quick estimate of his Big Five scores. Tests like these are often taken via social media, and the results are typically publicly posted by users. However, it is also possible to estimate Big Five traits using text mining of social media $\left(^{27}\right.$.

In the present case, the scores on the Big Five traits are given as percentages by Truity. In order to retrieve the records, we computed the percent scores on the Big Five traits, and computed the Euclidean distance to each of the records (i.e., the square root of the squared differences between each of the records in the dataset and the Truity data of the lead author), and converted these to z-scores. 
The R-script can be downloaded here: http://www.jolij.com/wp-

content/uploads/jolij vanmaeckelberghe koolhoven lorist $\mathrm{R}$ code.zip. A lower z-score indicates that a data record is more similar to the Big Five personality profile of the lead author as obtained via Truity ${ }^{28}$. Figure 1 shows the results: it is obvious that there is one record with a much lower Euclidean distance to the Big Five data of the author as measured via Truity than the others. We may therefore be reasonably sure that the author's ID is 30132 (this has been verified by the University of Amsterdam).

This is problematic given GPDR consideration 26: the principles of data protection should apply to any information concerning an identified or identifiable natural person. Pseudonymized personal data, as is the case here, should be considered to be information on an identifiable natural person if they can be attributed to a natural person by the use of additional information. It is doubtful whether this is the case in the example above, given that the we needed the author's Big Five scores in order to re-identify a record of his Big Five scores, but should this dataset have contained additional variables, this could have been problematic should the dataset have been publicly shared.

In the case of neurophysiological data this may be even more extreme. Evoked EEG responses, but background EEG as well, are highly specific for individuals, and can be used for personal identification with high accuracy ${ }^{29}, 30,31$. Since $\mathrm{FMRI}$ is even higher in dimensionality than EEG data, we may assume the same counts for BOLD responses. This means that effectively, this data cannot be de-identified once we know an individual's 'EEG signature', we can easily identify whether that person's records are present in the dataset or not.

In general we can state, the more variables are recorded in a dataset, the more likely it can be deidentified. However, the exact number of variables at which this becomes an issue cannot easily be determined. This strongly depends on what we may call a 'representational space' - the more unique combinations of values are possible within the recorded set of variables, the more likely it will be that a given individual can be identified by this combination. This, of course, depends on the distribution of the variables on interest within the population (i.e. when the database contains a record of the number of legs per participant, this number will typically be not very informative for identification purposes, unless it is not two, as participants with fewer than two legs will tend to be rare, and individuals with more than two legs even more exceptional). This means that for each dataset, one needs to consider whether 
the distribution of individual records in dataset's the representational space is such that individual records can be identified ${ }^{23}$.

It is important to note that identifiability of records in a dataset does not depend on that dataset alone, but also whether auxiliary information is available (i.e. information that can be obtained from other sources than the database an adversary may try to de-anonymize). A potential adversary who wants do re-identify a dataset published along with a research paper has several important sources of information. First, research papers typically detail the population from which the participants have been drawn. For the majority of psychology papers, participants are freshman or undergraduate students participating in exchange for course credit, and participate in multiple studies. In the latter case, the pool of participants can usually be easily identified, for example, all students from a given cohort. This becomes even easier when raw data is timestamped, and can be traced back to individual test sessions. Moreover with the rise of public data sharing, more and more datasets will be publicly available. This entails that it is likely datasets including data from the same participants will aggregate, given the sheer amount of studies using psychology undergraduates as research participant. If all these datasets are released, this means an adversary will have access to several additional records with which a dataset may be cross-referenced.

Of course one can argue that such auxiliary information is not easily available to an adversary without a direct connection to the research institution or population where the data was collected, and that one should not worry too much about this. However, when considering participants' rights, this is not the correct approach. The Dutch Council of State (the highest legal authority in the Netherlands on administrative law) has ruled that even if only a small number of individuals have sufficient information to easily identify records in a database, privacy protection legislation applies ${ }^{22}$.

In sum, potential de-anonymization of high dimensional datasets is a real problem, in particular when auxiliary information is openly available. The number of potential adversaries, no matter how small, is in principle irrelevant for discussions on participant privacy. Given that de-anonymization may be relatively easy, especially when an adversary has access to auxiliary information, and that in particular neuroimaging data is almost by definition traceable to individuals, public sharing of raw data poses a small, but very real risk of de-anonymization, and thus leaking of potentially sensitive information. Interestingly, this creates a direct conflict with the Declaration of Helsinki: according to Article 24 of the 
Declaration, researchers are obliged to take every precaution to safeguard privacy. Publicly sharing raw neuroimaging and possibly other high-dimensional data seems to be in direct violation of this Article ${ }^{18}$.

It should be noted here that in the previous discussion we assume that a researcher publishing data is well-aware of all the issues and technicalities regarding anonymization of a dataset. This, of course, does not need to be the case. As matter of fact, most psychologists are not information experts. This may lead to insufficient anonymization or to human error during uploading datasets. Lack of k-anonymity can, for example, be seen in the publicly shared datasets by the Australian Center on Quality of Life (see http://www.acqol.com.au/); the Belgium survey by De Maeyer, 2013, displays birthdates together with ethnicity, residence (located to a specific city and suburb in Belgium), number of children, and even the type of residence of the participant, but also contains records on chronic illness, mental health, and overall life satisfaction. These are easily obtainable records for any potential adversary, allowing for reidentification - many people post such information on social media like Facebook, for example. It is most likely that the researchers publishing this dataset are were not aware of proper anonymization protocols in order to ensure participant privacy - the dataset has been published on a private website of a group of researchers, and as far as we can see, has not been peer reviewed, possibly leading to this omission.

A final concern regarding privacy issues around public data sharing is that participants may become more wary of research participation and more cautious in their behavior during experiments. Given that de-anonymization may be a concern, and in the knowledge that online privacy is increasingly compromised, knowing that data will be published online can result in self-selection of participants, and more socially desirable behavior in experiments. Thus far, data on this is merely anecdotally, with some researchers reporting no drop in consent and research participation ${ }^{14}$, whereas others report a sharp drop in willingness to participate in experiment of which data may be published openly ${ }^{33}$. This does correspond to the prediction of the Dutch Autoriteit Gegevensbescherming ('Authority Personal Data') of the effect of openness in business. In business, 'privacy' will become the 'unique selling point', whereas openness will lead to a loss of customers. The same could be true for experiments as 'products' of research institutes.

\section{Consequences of re-identification of records}


Considering that de-anonymization of publicly shared data is a conceivable risk, what might the consequences of such a breach of confidentiality? Two main arguments are often mentioned in discussions on data confidentiality and participant anonymity: first, the number of potential adversaries is very small (and therefore the risk of re-identification as well). However, as mentioned before, legally this is not a valid argument - privacy protection applies regardless of the number of adversaries.

Second, most datasets in psychology consider relatively 'harmless' data, such as task performance scores, or reaction times - even in case of re-identification of such data, there would be not harm done to the participants. However, a 'neutral' measure such as EEG is increasingly used as a biomarker for traits such as mental health (for example, schizophrenia ${ }^{34}$; autism, ${ }^{35}$, or intelligence ${ }^{36}$ ) which obviously constitutes sensitive information. Moreover, other apparently 'innocent' measures, such as reaction times, or performance on a visual detection task, may be predictive of potentially sensitive traits as well: performance in a visual masking task may predict schizophrenia ${ }^{37}, 38$, proneness to false alarms in visual detection tasks correlates with belief in paranormal phenomena ${ }^{39}$, and performance on several visual search and detection tasks has been linked with religious beliefs ${ }^{40}$ - one study even claimed to have found lasting effects of previously held religious convictions on present perceptual performance in a simple visual detection task ${ }^{41}$.

One of course may argue that such inferences are invalid or at least problematic from a scientific viewpoint. Potential adversaries, though, may not necessarily keep up the same level of scientific rigor as scientists in order to draw (harmful) inferences about individuals. Let us not forget that under the present cultural and political climate, privacy is under increasing pressure, and that potential adversaries are going through increasingly greater lengths to uncover sensitive information about individuals. One only needs to look back at the 2016 presidential election cycle in the United States for ample examples it is imaginable that both Mrs. Clinton and Mr. Trump would have spared no efforts in attempts to deanonymize datasets in which any records of psychological data of the respective candidates may have been present. In the age of social media, even a relatively minor leak of information can have major consequences for an individual. There are countries in which leaking of personally sensitive information, such as sexual orientation or religious beliefs, can lead to criminal charges resulting in imprisonment or even capital punishment. It is therefore naïve to dismiss the potential for malicious de-anonymization of open data, given the potential serious consequences. 


\section{Participant and researcher autonomy, and public data sharing}

One final aspect to consider, although more theoretical than the previous sections, is autonomy - the moral principle that individuals should be able to make their own decisions about their actions ${ }^{42}$. This principle applies to the present issue as well. With regard to public data sharing, both participant and researcher have to make decisions regarding the consequences of publicly sharing data, not all of which are entirely clear at present.

Firstly, the participant as individual should not be considered a passive party in the enterprise of research: researcher and participant enter into a mutual contract stating the rights of the participant, and duties of the researcher, as laid out in the informed consent ${ }^{18}$. This entails that ultimately, the participant should decide upon the publication of her or his personal data. Usually, this is worded in the informed consent, which should of course explicitly mention that, in the case the researcher intends to publicly share data, (raw) data records which may be identified will be publicly shared. The GDPR (Article 4 (11) defines 'consent' as 'any freely given, specific, informed and unambiguous indication of the data subject's wishes by which he or she, by a statement or by a clear affirmative action, signifies agreement to the processing of personal data relating to him or her'. This consent is to be seen in the light of Article 7 GDPR and the principle of transparency. It requires that any information - also about the purpose and use of the data involved - addressed to the data subject be concise, easily accessible and easy to understand, and that clear and plain language and, additionally, where appropriate, visualisation be used. The data subject should be able to know and understand whether, by whom and for what purpose personal data relating to him or her are being collected (GDPR, consideration no 58).

However, it is an open question to what extent participants make such decisions carefully. Typically informed consents are boilerplate statements (or at least regarded as such). Based on research on how well (or poorly) people read terms and conditions of online services, it is conceivable that informed consents are hardly given any attention by research participants ${ }^{43}$. Can we safely assume that participants inform themselves of all possible consequences of giving informed consent for publicly sharing their data, if even researchers do not fully oversee the complexities of public data sharing (cf. the examples of insufficiently anonymized datasets provided above)? Without overseeing all possible consequences it is clear that it will be even harder to comply with the GDPR goals as a researcher in the future. The GDPR increases the quality of the information that should be provided to speak of 'informed consent'. When thinking of all possible consequences, one can imagine it will be hard to safeguard "a 
right to erasure, to be forgotten, to restriction of processing, to data portability, and to object when processing personal data for scientific research purposes" (consideration no 156).

Of course there are proper alternatives. The Harvard Personal Genome Project

(http://www.personalgenomes.org/), for example, makes personal genomic data and health status of participants publicly available. Genomic data is of course highly identifiable. However, a critical part in the enrollment process is an enrollment exam on consequences of participation (including the fact that data are shared in a non-anonymous manner) the participant needs to pass before she or he can participate. Similarly, neuroscientist Russ Poldrack recently published a longitudinal dataset of his own fMRI data ${ }^{44}$ - in this case, the publicly shared data concerns a named individual.

One could argue, though, that participants ought to be protected from themselves, and that given the potential harm de-identification can bring, in particular when health or health-related information is concerned, one should not even offer the option of having such data publicly shared. Whether such a viewpoint is considered to be patronizing strongly depends on one's social and cultural environment, of course. There is considerable debate on this particular aspect in the legal profession. Article 7 (3) has tried to solve this debate by giving the data subject 'the right to withdraw his or her consent at any time'. However, the withdrawal of consent does not affect the lawfulness of processing based on consent before its withdrawal. And, where data are further processed and used by other scientists before the withdrawal of consent, the protective effect of this Article is minimal.

Researchers, too, will need to make decisions about whether to publicly share data or not. Within psychological science, there is an increasing tendency towards transparency, with several high-profile researchers pushing this transform, and engaging in public sharing themselves (cf. ${ }^{44}$ ). Some of these researchers feel by far most data can and should be publicly shared ${ }^{11}$. However, as we have shown in this paper, this is not as straightforward as it seems. Potential ethical and legal problems are lurking at each stage of data preparation and publication, and a lot is still unclear with regard to responsible public data sharing. Processing of data is lawful in the sense of Article 6 (1) (f) when the data processing is necessary for the purposes of the legitimate interests pursued by the controller or by a third party, except where such interests are overridden by the interests or fundamental rights and freedoms of the data subject which require protection of personal data, in particular where the data subject is a child. Researchers, as controllers, are under the obligation of balancing their "legitimate interests" with the 
fundamental rights and freedoms of the data subject. One does wonder, whether science should be improved by sharing data at the risk of research participants rights, whereas one could also improve the quality of research in other ways, thinking about the rewarding system in science in general.

\section{Conclusion}

We strongly believe in the values of open and transparent science, and we applaud the recent initiatives that have been launched to improve the quality of science. However, given that we as psychologists and cognitive neuroscientists work with human subject data, we should be extremely careful when being open with our data. It is important to realize that meeting the conditions for safe and responsible public data sharing is deceivingly difficult. Being an ethical scientist does not only mean we should adhere to the highest possible scientific standards, but also that we have a responsibility to the people who enable us to do our work in the first place: our participants. Publicly sharing their data is something we should not take for granted, but carefully deliberate for every single dataset.

Moreover, it seems the present discussion primarily focuses on the moral duty of sharing data for the sake of science, and finding ways of pushing public data sharing despite legal and/or ethics limitations, or shifting legal and ethical norms, in particular regarding participant privacy. It is true that opinions with regard to legal and ethical restrictions vary from researcher to researcher, and even from lawyer to lawyer. However, we strongly urge researchers to adhere to the strictest interpretation of ethical and legal constrictions, for safeguarding one's own legal position, but most importantly for the obvious protection of participant interests.

As a final remark, though, we would like to point out the following: it is clear that the open science movement has irrevocably changed science for the better, and put it on a course towards more transparency. However, it is important that we ask ourselves the question how come that our science went from being transparent and collective to the competitive and opaque enterprise it appears to be nowadays. Why do researchers refuse to share their data, and materials? To push open data and open science without addressing this deeper question will on the long term not lead to a better science. One of the reasons researchers may be reluctant to share data is because they do not feel safe when doing so, for several of the personal reasons we mentioned above. In the present, highly competitive climate, one's reputation, funding and thus job security depends on the number of publications and grants one gets in. Data is at the core of such publications, and sharing that data makes one vulnerable. In order to 
truly inspire a lasting change in openness values, we need to look further than simply pushing open science, but we will have to provide all researchers with a safer haven - an aspect of the science reform movement that we feel should get as much attention as openness ${ }^{45}$. 


\section{References}

1. Bem, D. J. Feeling the future: experimental evidence for anomalous retroactive influences on cognition and affect. J. Pers. Soc. Psychol. 100, 407-425 (2011).

2. Levelt Committee. Flawed Science: the fraudulent research practices of social psychologist Diederik Stapel. (University of Tilburg, 2012).

3. loannidis, J. P. A. Why most published research findings are false. PLoS Med. 2, e124 (2005).

4. Open Science Collaboration. PSYCHOLOGY. Estimating the reproducibility of psychological science. Science 349, aac4716 (2015).

5. Button, K. S. et al. Power failure: why small sample size undermines the reliability of neuroscience. Nat. Rev. Neurosci. 14, 365-376 (2013).

6. Wicherts, J. M., Bakker, M. \& Molenaar, D. Willingness to share research data is related to the strength of the evidence and the quality of reporting of statistical results. PloS One 6, e26828 (2011).

7. Wicherts, J. M., Borsboom, D., Kats, J. \& Molenaar, D. The poor availability of psychological research data for reanalysis. Am. Psychol. 61, 726-728 (2006).

8. Vanpaemel, W., Vermorgen, M., Deriemaecker, L. \& Storms, G. Are We Wasting a Good Crisis? The Availability of Psychological Research Data after the Storm. Collabra Psychol. 1, (2015).

9. Naik, G. Peer-review activists push psychology journals towards open data. Nat. News 543, 161 (2017).

10. Eich, E. Business Not as Usual. Psychol. Sci. 25, 3-6 (2014).

11. Morey, R. D. et al. The Peer Reviewers' Openness Initiative: incentivizing open research practices through peer review. R. Soc. Open Sci. 3, 150547 (2016).

12. Kidwell, M. C. et al. Badges to Acknowledge Open Practices: A Simple, Low-Cost, Effective Method for Increasing Transparency. PLOS Biol. 14, e1002456 (2016). 
13. Nichols, T. E. et al. Best practices in data analysis and sharing in neuroimaging using MRI. Nat. Neurosci. 20, 299-303 (2017).

14. Rouder, J. N. The what, why, and how of born-open data. Behav. Res. Methods 48, 1062-1069 (2016).

15. de Wolf, V. A., Sieber, J. E., Steel, P. M. \& Zarate, A. O. Part II: HIPAA and Disclosure Risk Issues. IRB Ethics Hum. Res. 28, 6-11 (2006).

16. Munafò, M. R. et al. A manifesto for reproducible science. Nat. Hum. Behav. 1, 21 (2017).

17. de Vey Mestdagh, C., Dijkstra, J. J., Paapst, M. H., Bennigsen, I. \& van Zuijlen, T. IT voor Juristen: recht zoeken, recht vinden. (Stichting Recht \& ICT, 2016).

18. World Medical Association Declaration of Helsinki: Ethical Principles for Medical Research Involving Human Subjects. JAMA 310, 2191-2194 (2013).

19. Koninkrijksrelaties, M. van B. Z. en. Wet medisch-wetenschappelijk onderzoek met mensen. (2017).

20. Regulation (EU) 2016/679 of the European Parliament and of the Council of 27 April 2016 on the protection of natural persons with regard to the processing of personal data and on the free movement of such data, and repealing Directive 95/46/EC (General Data Protection Regulation). Off. J. Eur. Union L119, 1-88 (2016).

21. El Emam, K., Jonker, E., Arbuckle, L. \& Malin, B. A systematic review of re-identification attacks on health data. PloS One 6, e28071 (2011).

22. ECLI:NL:RVS:2012:BY2508. (2012).

23. Sweeney, L. K-anonymity: A Model for Protecting Privacy. Int J Uncertain Fuzziness Knowl-Based Syst 10, 557-570 (2002).

24. Narayanan, A. \& Shmatikov, V. Robust De-anonymization of Large Sparse Datasets. in Proceedings of the 2008 IEEE Symposium on Security and Privacy 111-125 (IEEE Computer Society, 2008). doi:10.1109/SP.2008.33 
25. Smits, I. A. M., Dolan, C. V., Vorst, H. C. M., Wicherts, J. M. \& Timmerman, M. E. Cohort differences in Big Five personality factors over a period of 25 years. J. Pers. Soc. Psychol. 100, 1124-1138 (2011).

26. Smits, I., Dolan, C., Vorst, H., Wicherts, J. \& Timmerman, M. Data from 'Cohort Differences in Big Five Personality Factors Over a Period of 25 Years'. J. Open Psychol. Data 1, (2013).

27. Wald, R., Khoshgoftaar, T. \& Sumner, C. Machine prediction of personality from Facebook profiles. in 2012 IEEE 13th International Conference on Information Reuse Integration (IRI) 109-115 (2012). doi:10.1109/IRI.2012.6302998

28. Marco, V. R., Young, D. M. \& Turner, D. W. The Euclidean distance classifier: an alternative to the linear discriminant function. Commun. Stat. - Simul. Comput. 16, 485-505 (1987).

29. De Vico Fallani, F., Vecchiato, G., Toppi, J., Astolfi, L. \& Babiloni, F. Subject identification through standard EEG signals during resting states. Conf. Proc. Annu. Int. Conf. IEEE Eng. Med. Biol. Soc. IEEE Eng. Med. Biol. Soc. Annu. Conf. 2011, 2331-2333 (2011).

30. Hema, C. R. \& Osman, A. A. Single trial analysis on EEG signatures to identify individuals. in 20106 th International Colloquium on Signal Processing its Applications 1-3 (2010). doi:10.1109/CSPA.2010.5545313

31. Tangkraingkij, P., Lursinsap, C., Sanguansintukul, S. \& Desudchit, T. Personal Identification by EEG Using ICA and Neural Network. in Computational Science and Its Applications - ICCSA 2010 419-430 (Springer, Berlin, Heidelberg, 2010). doi:10.1007/978-3-642-12179-1_35

32. De Maeyer, J. Single cross sectional survey report, Ghent. (2013).

33. Jolij, J. The Open Data Pitfall II - Now With Data. Belief, Perception, and Cognition Lab (2015).

34. Haigh, S. M., Coffman, B. A. \& Salisbury, D. F. Mismatch Negativity in First-Episode Schizophrenia: A Meta-Analysis. Clin. EEG Neurosci. 48, 3-10 (2017). 
35. Vandenbroucke, M. W. G., Scholte, H. S., van Engeland, H., Lamme, V. A. F. \& Kemner, C. A neural substrate for atypical low-level visual processing in autism spectrum disorder. Brain J. Neurol. 131, 1013-1024 (2008).

36. Jolij, J. et al. Processing speed in recurrent visual networks correlates with general intelligence. Neuroreport 18, 39-43 (2007).

37. Chkonia, E. et al. The shine-through masking paradigm is a potential endophenotype of schizophrenia. PloS One 5, e14268 (2010).

38. Chkonia, E. et al. Patients with functional psychoses show similar visual backward masking deficits. Psychiatry Res. 198, 235-240 (2012).

39. Krummenacher, P., Mohr, C., Haker, H. \& Brugger, P. Dopamine, paranormal belief, and the detection of meaningful stimuli. J. Cogn. Neurosci. 22, 1670-1681 (2010).

40. Colzato, L. S., Hommel, B. \& Shapiro, K. L. Religion and the attentional blink: depth of faith predicts depth of the blink. Front. Psychol. 1, 147 (2010).

41. Colzato, L. S. et al. God: Do I have your attention? Cognition 117, 87-94 (2010).

42. Christman, J. Autonomy in Moral and Political Philosophy. in The Stanford Encyclopedia of Philosophy (ed. Zalta, E. N.) (Metaphysics Research Lab, Stanford University, 2015).

43. Obar, J. A. \& Oeldorf-Hirsch, A. The Biggest Lie on the Internet: Ignoring the Privacy Policies and Terms of Service Policies of Social Networking Services. (Social Science Research Network, 2016).

44. Poldrack, R. A. et al. Long-term neural and physiological phenotyping of a single human. Nat. Commun. 6, 8885 (2015).

45. Benedictus, R., Miedema, F. \& Ferguson, M. W. J. Fewer numbers, better science. Nat. News 538, 453 (2016). 


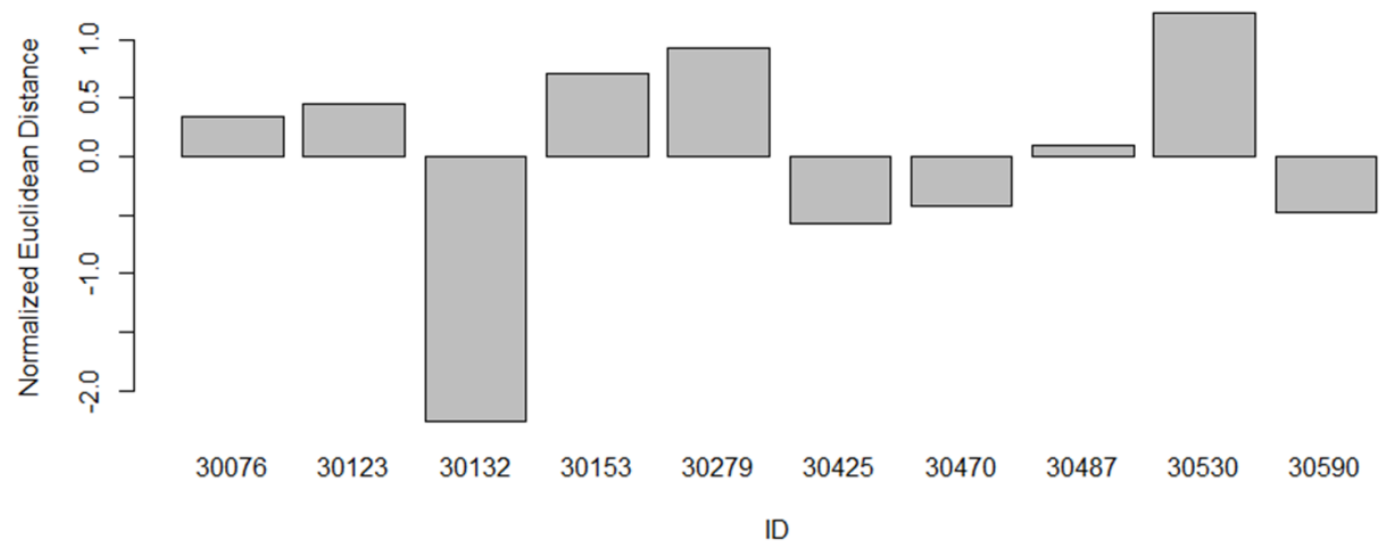

Figure 1. Re-identification of the Big Five dataset using normalized Euclidean distances (i.e., the z-score over all 10 records). The $\mathrm{Y}$-axis gives the normalized Euclidean distance; lower scores indicate that a data record is more similar to the target record. It is obvious that record 30132 is most similar to the target record, and thus most likely the identifier we are looking for. 